\title{
Avaliação do perfil das vítimas de fraturas de fêmur atendidos em um hospital de urgência e emergência na amazônia ocidental brasileira
}

Assessment of the profile of victims of femur fractures treated at an urgent and emergency hospital in the Brazilian western Amazon

Evaluación del perfil de las víctimas de fracturas de fémur atendidas en un hospital de urgencia y emergencia en la Amazonía occidental brasileña

Recebido: 09/01/2022 | Revisado: 05/02/2022 | Aceito: 06/02/2022 | Publicado: 11/02/2022

Ennely Mendonça Gutzeit

ORCID: https://orcid.org/0000-0002-0566-1276

Centro Hospital João Paulo II, Brasil

E-mail: ennely@gmail.com

Thiago Vaz Lopez

ORCID: https://orcid.org/0000-0002-3192-1908

Universidade Federal do Acre, Brasil

E-mail: thiagovlopes@hotmail.com

Silvecler Cortijo de Campos

ORCID: https://orcid.org/0000-0003-0522-0814

Hospital de Base Ary Pinheiro, Brasil

E-mail: silvinhocortijo@hotmail.com

Breno de Oliveira Chagas Barreto

ORCID: https://orcid.org/0000-0003-4952-0288

Centro Universitário São Lucas, Brasil

Email: Kenjiolive@gmail.com

Camila Wehbe

ORCID: https://orcid.org/0000-0002-7770-0350 Centro Universitário São Lucas, Brasil

E-mail: wehbefarma@gmail.com

Iara Vaz Lopes

ORCID: https://orcid.org/0000-0003-0974-4615

Centro Universitário Aparício Carvalho, Brasil E-mail: lopesiaravaz@hotmail.com

Josiel Neves da Silva

ORCID: https://orcid.org/0000-0002-7383-3631 Centro Universitário São Lucas, Brasil E-mail: josiel.nsilva@gmail.com

João Gustavo da Silva Garcia de Souza

ORCID: https://orcid.org/0000-0002-5899-2490

Centro Universidade Aparício Carvalho, Brasil

E-mail: joaogarciadesouza27@gmail.com

Juliana Costa Rodrigues

ORCID: https://orcid.org/0000-0003-0712-7584

Centro Universitário São Lucas, Brasil

E-mail: julianamedvet_@ hotmail.com

Raissa Santos Reimann

ORCID: https://orcid.org/0000-0001-7860-8727

Centro Universitário São Lucas, Brasil

E-mail: raissareimann@hotmail.com

Igor Mansur Muniz

ORCID: https://orcid.org/0000-0003-0863-6647

Universidade Federal de Rondônia, Brasil

E-mail: igor.mansur@unir.br

\section{Resumo}

As fraturas do fêmur apresentam maior morbimortalidade em todos os níveis de atenção à saúde. Dessa forma, as quedas em idosos são bastante frequentes pois, acarretam na diminuição da capacidade funcional e consequências graves para esses pacientes. Os acidentes de trânsito são relevantes, acometendo mais, os adultos jovens do sexo masculino sendo a imprudência a principal causa desses acidentes. Avaliou-se o perfil das vítimas de fraturas de fêmur atendidos em um hospital de urgência e emergência de Porto Velho/RO, através da estratificação dos dados apresentados por sexo, faixa etária, lado acometido e mecanismo de trauma. A metodologia deste estudo foi realizada através de dados coletados a 
partir de prontuários médicos, deliberados pelo sistema HOSPUB. Os resultados, foram avaliados o quantitativo de admissões traumáticas pela ortopedia no período de janeiro a abril do ano de 2021, totalizando 2.043 pacientes atendidos na sala vermelha e ambulatório, desse total 63 são de fratura de fêmur 3,08\% (63/2.043), sendo a distribuição desses nos meses; janeiro 41,2\% (26/63), fevereiro 17,4\% (11/63), março 25,3\% (16/63) e abril 15,8\% (10/63). Avaliou-se a variável sexo, dentre os pacientes atendidos, observou-se um predomínio do sexo masculino na faixa etária de 21-49 anos 30,5\% (19/63) estando equivalente quando comparado com outros resultados, sendo esse o sexo mais acometido por acidentes e violências. Conclui-se, que o perfil das vítimas com fratura de fêmur tem predomínio no sexo feminino quando tratando de idosos, onde a principal causa são as quedas. Já os adultos jovens a maior prevalência e do sexo masculino tendo como o principal mecanismo de trauma os acidentes de motocicleta.

Palavras-chave: Fraturas de fêmur; Quedas; Acidente de trânsito.

\begin{abstract}
Femur fractures have higher morbidity and mortality at all levels of health care. Thus, falls in the elderly are quite frequent as they lead to a decrease in functional capacity and serious consequences for these patients. Traffic accidents are relevant, affecting more young male adults, with recklessness being the main cause of these accidents. The profile of victims of femur fractures treated at an urgent and emergency hospital in Porto Velho/RO was evaluated, through the stratification of the data presented by sex, age group, affected side and trauma mechanism. The methodology of this study was carried out through data collected from medical records, deliberated by the HOSPUB system. The results were evaluated the number of traumatic admissions by orthopedics in the period from January to April of the year 2021, totaling 2,043 patients treated in the red room and outpatient clinic, of this total 63 are femur fracture 3.08\% (63/2,043) , being the distribution of these in the months; January 41.2\% (26/63), February $17.4 \%$ (11/63), March 25.3\% (16/63) and April 15.8\% (10/63). The gender variable was evaluated, among the patients seen, there was a predominance of males in the age group of 21-49 years $30.5 \%$ (19/63) being equivalent when compared to other results, this being the most affected by accidents and violence. It is concluded that the profile of victims with femur fractures is predominant in females when treating the elderly, where the main cause is falls. The highest prevalence among young adults is male, with motorcycle accidents as the main trauma mechanism.
\end{abstract}

Keywords: Femur fractures; Falls; Traffic accident.

\title{
Resumen
}

Las fracturas de fémur tienen mayor morbilidad y mortalidad en todos los niveles de atención de la salud. Así, las caídas en los ancianos son bastante frecuentes ya que conllevan una disminución de la capacidad funcional y graves consecuencias para estos pacientes. Los accidentes de tránsito son relevantes, afectando más a adultos jóvenes varones, siendo la imprudencia la principal causa de estos accidentes. Se evaluó el perfil de las víctimas de fracturas de fémur atendidas en un hospital de urgencia y emergencia de Porto Velho/RO, a través de la estratificación de los datos presentados por sexo, grupo de edad, lado afectado y mecanismo del trauma. La metodología de este estudio se realizó a través de datos recolectados de prontuarios, deliberados por el sistema HOSPUB. Los resultados fueron evaluados el número de ingresos traumáticos por ortopedia en el periodo de enero a abril del año 2021, totalizando 2.043 pacientes atendidos en sala roja y ambulatorio, de este total 63 son fractura de fémur 3,08\% (63/2.043), siendo la distribución de estos en los meses; enero 41,2\% (26/63), febrero 17,4\% (11/63), marzo 25,3\% (16/63) y abril 15,8\% (10/63). Se evaluó la variable género, entre los pacientes atendidos hubo predominio del sexo masculino en el grupo de edad de 21 a 49 años 30,5\% (19/63) siendo equivalente al comparar con otros resultados, siendo este el más afectado por accidentes y violencia. Se concluye que el perfil de víctimas con fracturas de fémur es predominante en el sexo femenino cuando se trata al adulto mayor, donde la principal causa son las caídas. La mayor prevalencia entre adultos jóvenes es del sexo masculino, siendo los accidentes de motocicleta el principal mecanismo traumático.

Palabras clave: Fracturas de fémur; Caídas; Accidente de trânsito.

\section{Introdução}

A priori, a longevidade condicionada à transição demográfica se tornou comum em vários países. Sendo assim, no ano de 2060 estima-se, que terá um aumento de 38 milhões de brasileiros acima dos 60 anos (Oliveira et al., 2020). Nesse sentido, os fatores biológicos sociais, econômicos, além de causas externas e doenças contribuem para o envelhecimento. Estando assim, associadas à diminuição significativa da capacidade funcional do idoso (Soares et al., 2014). Dessa forma, as quedas em idosos são bastante frequentes causando problemas psicológicos e sociais e consequências graves para esses pacientes (Maia et al., 2011).

As doenças mais prevalentes entre os idosos encontra-se a fratura do fêmur, sendo o sexo feminino o mais acometido, pois a falta do hormônio estrogênio causa fragilidade óssea (Machado et al., 2012). Além disso, as fraturas do fêmur geram 
impactos onerosos para a saúde devido a longos períodos de hospitalização e complicações, para esses pacientes (Santos Júnior et al., 2021).

Diante do exposto, fraturas osteoporóticas são mais significativas nas mulheres a partir dos 50 anos, sendo sua incidência proporcional ao aumento da idade, menopausa precoce, histórico familiar, histórico prévio de fratura, Diabetes Mellitus tipo 1 e 2 e o baixo consumo de cálcio (Santos et al., 2021). Assim, as fraturas do fêmur apresentam maior morbimortalidade em todos os níveis de atenção à saúde (Bracco et al., 2009). Assim, medidas preventivas e o controle das complicações e doenças associadas, poderiam reduzir os agravos, a mortalidade e a incapacidade funcional desses pacientes (Borges et al., 2012).

Os acidentes de trânsito apresentam um grave problema de saúde pública (Rios et al., 2019). Dessa forma, a média de mortes no mundo chega a 1,24 milhão nas estradas (Peden, 2005). Além disso, os acidentes de trânsito prejudicam o setor econômico, sobretudo nos países em desenvolvimento, em virtude do afastamento das atividades laborais (Araujo et al, 2021).

A incidência de traumas de alta energia, vem acarretando o aparecimento de fraturas diafisárias de difícil tratamento (Pires res, et al., 2010). Esse grupo apresenta mais homens no qual, são jovens economicamente ativos, que vão esta sujeitos à redução cirúrgica das fraturas do fêmur (Dias et al.,2021). Com isso, as lesões acarretam a qualidade de vida dos pacientes, com fraturas de fêmur dificultando suas atividades laborais e onerando os serviços públicos (Rocha et al., 2020).

Com isso, o presente estudo tem como objetivo avaliar o perfil das vítimas de fraturas de fêmur atendidos em um hospital de urgência e emergência de Porto Velho/RO.

\section{Metodologia}

Os dados deste estudo foram coletados a partir de prontuários médicos, deliberados pelo sistema HOSPUB em um Hospital de urgência e emergência de porto velho/RO. Sendo realizado no mês de dezembro do ano de 2021 , tendo como referência os atendimentos, do mês de janeiro a abril do mesmo ano, realizados pelo setor de ortopedia e traumatologia.

A composição da amostra se deu pelo quantitativo de pacientes atendidos nesse período, para participar da pesquisa os pacientes foram vítimas de traumas dando entrada direto para o referido setor ou por outra especialidade médica, com posterior encaminhamento interno para a ortopedia e traumatologia.

Os dados coletados dos prontuários foram digitados em planilhas do programa Microsoft Office Excel® para organização e padronização dos dados e posteriormente exportados para um segundo programa denominado de Statistic $®$, sendo neste avaliado medias, prevalência, mediana e desvio padrão, e a junção dos dados gerados por ambos os programas, apresentados em forma de tabelas e gráficos.

Todos os preceitos éticos nacionais e internacionais foram respeitados, sendo essa pesquisa submetida e autorizada pelo comitê de ética em pesquisa e experimentação em humanos, CEP 4.875.244 de 30 de Julho de 2021, sendo ainda observando e seguido a Resolução 196/96 do Conselho Nacional de Saúde.

\section{Resultados}

Foram avaliados o quantitativo de admissões traumáticas pela ortopedia em um hospital de pronto atendimento na cidade de Porto Velho-RO, revelando o perfil dessas vítimas, bem como algumas variáveis. Os atendimentos realizados de janeiro a abril do ano de 2021, totalizando 2.043 pacientes atendidos na sala vermelha e ambulatório do hospital de referência em ortopedia e traumatologia na cidade de Porto Velho-Rondônia, desse total 63 são de fratura de fêmur 3,08\% (63/2.043), sendo a distribuição desses nos meses; janeiro 41,2\% (26/63), fevereiro 17,4\% (11/63), março 25,3\% (16/63) e abril 15,8\% (10/63). 
Quando avaliado o número de atendimentos frente a variável sexo, foi constatado um predomínio do masculino 54\% (34/63) para 46\% do feminino (29/63), sendo a faixa etária masculina de maior prevalência a de 21-30 anos com 12,6\% (8/63) e do sexo feminino de 71-80 anos com 15,8\% (10/63). Conforme demonstrado na Tabela-1.

Tabela 1 - Distribuição das vítimas de traumas fêmur, atendidas na sala vermelha de um hospital de pronto atendimento na cidade de Porto Velho-RO, em porcentagem para o sexo e faixa etária.

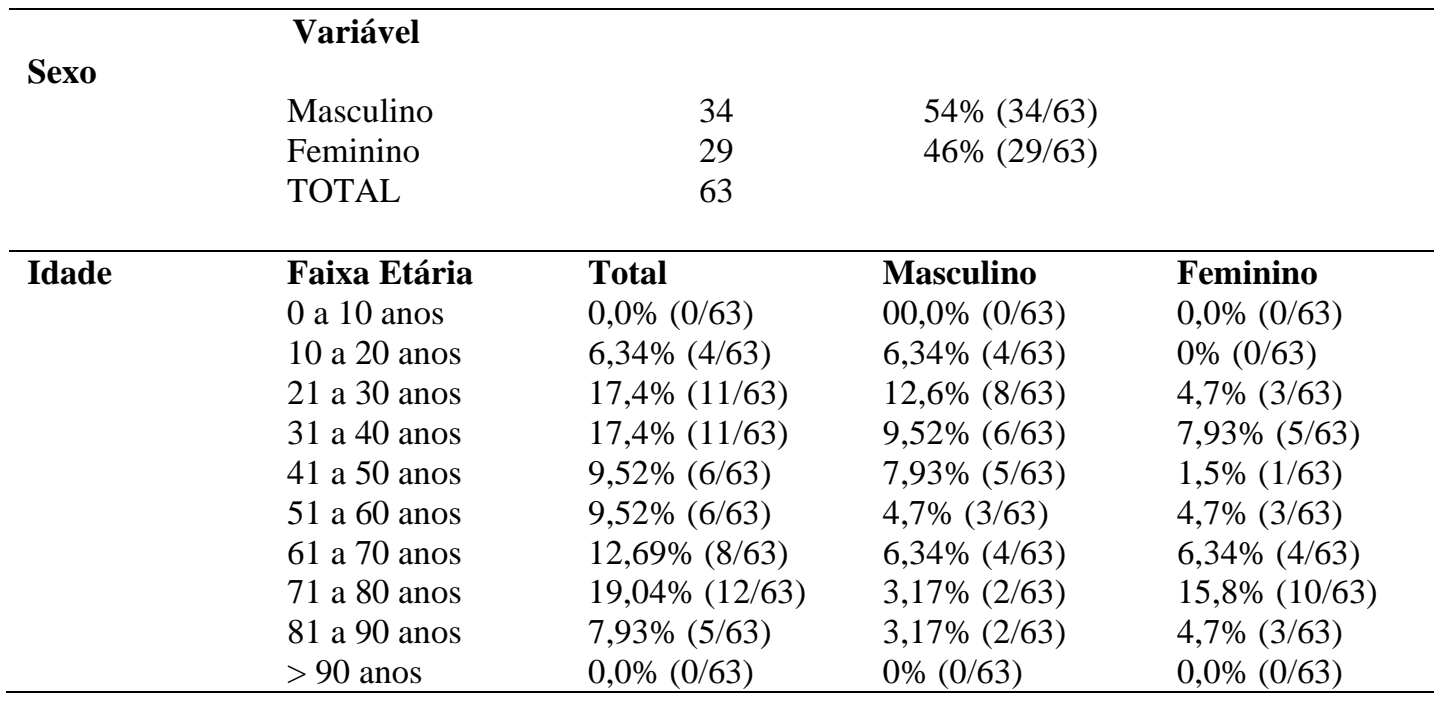

Fonte: Autores.

Quanto ao lado de acometido houve um predomínio do lado direito 52,3\% (33/60), quando comparado ao esquerdo 44,4\% (28/63). Em relação ao mecanismo do trauma acidente trânsito-moto representou 44,4\% (28/63) dos casos, seguido de acidente doméstico (queda) 31,7\% (20/63), causas diversas 17,4\% (11/63), acidente de trabalho 1,5\% (1/63), acidente de trânsitobicicleta $1,5 \%$ (1/63) e acidente de arma de fogo 1,5\% (1/63), demonstrado no Gráfico abaixo.

Gráfico 1- Quantitativo de pacientes atendidos pela ortopedia e traumatologia, em um hospital de pronto atendimento na cidade de Porto Velho-RO, demonstrando as diferentes origens do trauma.

\section{Mecanismo do Trauma}

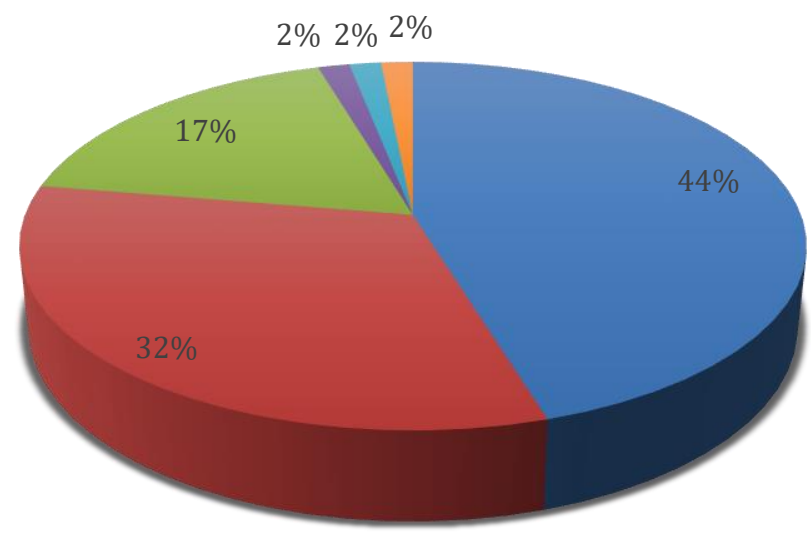

口. Ac. Trânsito-moto

Ac. Doméstico (queda)

- Causas diversas

Ac. De trabalho

Ac. Trânsito-bicicleta

Ac. Arma de fogo

Fonte: Autores. 
Dentre os pacientes internados quatro deles perduraram por mais tempo devido a complicações, sendo que um deles já era nefropata com histórico de dialise por 1 ano e 6 meses, permanecendo internado por 22 dias aguardando avaliação da nefrologia. Uma segunda paciente, idosa com história de aneurisma, hipertensão arterial e diabetes melitus permaneceu internada por 5 dias, até que se obtivesse estabilidade hemodinâmica para a transferência, para um hospital de alta complexidade. Um terceiro com história de politraumas e artrose avançada, vítima de acidente de trânsito permanecendo internado por 4 dias aguardando transferência. Quarto paciente com complicações foi um idoso com histórico de retardo mental desde infância, com diabetes melitus sem tratamento, apresentando hepatopatia e nefropatia internado há 4 dias aguardando transferência.

\section{Discussão}

Nesse estudo, quando avaliado a variável sexo dentre os pacientes atendidos, observou-se um predomínio do sexo masculino na faixa etária de 21-49 anos 30,5\% (19/63) estando equivalente quando comparado por aos resultados de Conceição et al., (2016) ao encontrar 38,87\%, sendo esse o sexo mais acometido por acidentes e violências. Já para o feminino a maior prevalência da faixa etária está entre 70 anos ou mais com uma porcentagem 20,5\%, ainda quando comparado a esse mesmo autor, observou discrepância dos resultados (75,53\%), embora essa diferença grande de valores, concordamos que essa e a faixa etária com maior prevalência para mulheres, tendo elas o principal mecanismo de fratura a queda da própria altura.

Nesse sentido, o principal fator determinante na ocorrência ou não de uma fratura é a qualidade óssea. Mulheres após a menopausa, tendem a baixos níveis de estrogênio, responsável pela manutenção da massa óssea, tornando os ossos menos resistentes a impactos (Rocha et al., 2020). Associado a isso o sedentarismo, histórico familiar de osteoporose, ciclos menstruais irregulares, ausência de gestação e uso de alguns medicamentos (corticosteroides, anticonvulsivantes, hidróxido de alumínio, diuréticos, anti-inflamatórios) contribuem para uma rápida degeneração óssea (Araújo et al., 2020).

Segundo relatado por Morais et al., (2016), os mecanismos de trauma devido a mortalidade fazem dessas fraturas um problema de saúde pública, estando dentre os principais mecanismos os de acidentes de trânsito, compreendendo 50\% deles, porcentagem essas equivalentes aos apresentados nessa pesquisa (44,4\%) para os acidentes automobilístico.

Nesse contexto, diversos fatores colaboram com essa realidade, os acidentes de trânsito relacionados às motocicletas geram inúmeros problemas de saúde pública para o país. Com isso, o aumento de veículos em circulação, além da falta de leis mais severas, a impunidade, a falta de fiscalização adequada, a idade avançada da frota e a falta de estrutura em vias públicas geram problemas relacionados à sinalização (Junior et al., 2021), e a reabilitação para esses pacientes e falta de alocação para o mercado de trabalho geram despesas para o Estado.

Em relação aos acidentes doméstico, a queda da própria altura representa uma porcentagem de 31,7\% nessa pesquisa, e quando comparado com Muniz et al., (2007), se demonstra bem inferior perto dos 89,89\% por ele demonstrado. Dessa forma, esse tipo de fratura poderia ser evitado por medidas simples e de baixo custo, como a prevenção e tratamento da osteoporose, de déficits oftalmológicos e modificações nas condições de vida cotidiana e eliminando obstáculos que eventualmente possam causar acidentes.

Diante o exposto, o principal fator responsável pelo aumento na incidência de fratura de fêmur na faixa etária acima dos 60 anos é a osteoporose, estima-se que um terço das mulheres da raça branca com idade superior a 65 anos tenha osteoporose e $30 \%$ delas sofram ao menos uma queda por ano. Com isso, essas fraturas atingem principalmente mulheres idosas, considerada um dos maiores problemas da saúde pública no país (Soares et al., 2015).

Nessa pesquisa, os acidentes de trabalho representam 1,5\% das fraturas de fêmur já quando comparado ao estudo de Morais et al., (2016), esse valor está abaixo dos por ele apresentado que foi de 6\%, necessitando assim esses de reabilitação para realocação de suas funções laborais. 
Nesse estudo, as lesões por projétil de arma de fogo (PAFs) representam 1,5\% das fraturas de fêmur com acometimento do sexo masculino, quando comparado aos resultados demonstrados por BAUMFELD et al., (2019) por esse mesmo mecanismo em que foi demonstrado a porcentagem de 94,4\%, para essa mesma causa, porém ele trabalhou com ao acometimento dessas diferentes partes ósseas; coluna vertebral, fêmur, tíbia, fíbula, mão e antebraço. Já o autor Morais et al., (2016), que trabalhou dados específicos para fratura de fêmur também por esse mecanismo, apresentou um número médio (1\%) equivalente ao encontrado nessa pesquisa.

Por fim, a incidência de fratura de fêmur vem aumentando na atualidade, a qualidade de vida desses pacientes devidas as doenças como osteoporose ficam comprometidas no qual, atinge mais o sexo feminino pois, ocorre a redução do hormônio estrogênio acarretando a fragilidade óssea.

Dessa forma, outra consequência são as quedas que afetam os idosos que acabam sendo prejudicados e dificultando suas atividades laborais. Além disso, os acidentes de trânsito também representam um quantitativo relevante atingindo principalmente homens na faixa etária de 21-49 anos tem uma maior prevalência de acordo com os dados coletados do presente estudo.

Com isso, a morbimortalidade desses pacientes é alta e o impacto na saúde pública acaba sendo oneroso para o Estado pois, o aumento do tempo de internação e complicações para esses pacientes contribuem para tal situação. Assim, os resultados deste estudo demonstram direcionamento para estratégias de prevenção para políticas públicas bem como campanhas midiáticas na prevenção de quedas e conscientização no trânsito seria boa medidas para esses pacientes.

\section{Conclusão}

Conclui-se, que os resultados obtidos ao longo do trabalho, foram avaliar o perfil das vítimas com fratura de fêmur, tendo em vista a maior prevalência no sexo feminino quando tratando de idosos, associado a uma das principais consequências, graves que são as quedas. Já os adultos jovens o predomínio foi do sexo masculino sendo o principal mecanismo de trauma os acidentes de motocicleta

\section{Referências}

Araújo, D. D. C.; Almeida, C. P.; Santana, L. R. P.; Santos, A. D. D.; Lima, S. V. M. A.; Araújo, K. C. G. M. D.; Alves, J. A. B.; Filho, V. J. D. S. \& Vaez, A. C. (2021). Fatores preditores e qualidade de vida das vítimas de trauma por acidentes de trânsito; Research, Society and Development, v. 10, n. 5, e 0410514576 .

Araújo, L. B.; Garces, T. S.; Sousa, G. J. B.; Moreira, T. M. M.; Pereira, M. L. D.; Damasceno, L. L. V.; Gomes, I. M.; Gomes, L. A. (2020). Tendência de hospitalizações por fratura de fêmur no Brasil: uma série temporal. Braz. J. of Develop, Curitiba, v. 6, n.5, p.28499-28510.

Baumfeld, D.; Brito, A. S. P.; Torres, M. S.; Prado, K. L.; Andrade, M. A. P.; Campos, T. V. O. (2020). Fraturas causadas por armas de fogo: Epidemiologia e taxa de infecção. Minas Gerais, Belo Horizonte. Rev Bras Ortop 2020;55(5):625-628.

Borges, A. E. A.; Araújo, K. M. B.; Stolt, L. R. O. G.; Ferreira, J. J. A. (2012). Caracterização das Fraturas do Fêmur em Pacientes de um Hospital de Emergência e Trauma em João Pessoa-PB no Período de 2008/2009. Revista Brasileira de Ciências da Saúde. Volume 16 Número 4 Páginas $507-516$.

Bracco, O.; Fortes E.; Raffaelli. M. P.; Araújo, D. V.; Santili. C.; Lazaretti, C. M. (2009). Custo hospitalar para tratamento da fratura aguda do fêmur por osteoporose em dois hospitais-escola conveniados ao Sistema Único de Saúde. J Bras Econ Saúde, 1(1):3-10.

Conceição, A. M.; Filho, F. C. G.; Dias, J. P. (2016). Internações por fraturas de fêmur em salvador, Bahia. Revista Baiana de Saúde Pública v. 40, n. 2, p. 298314 abr./jun.

Dias, D. F.; \& Gonçalves, S. J. C. (2021). Falhas em implantes de quadril. Pesquisa, Sociedade e Desenvolvimento, [S. 1.], v. 10, n. 11, pág. e357101119668.

Fonseca, F. F.; Pereira, L. S. M.; Arantes, P. M. M. (2012). Fatores associados a desfechos clínicos em idosos com fratura proximal de fêmur atendidos em hospital escola. Belo Horizonte - MG. Artigo Original. Sociedade Brasileira de Geriatria e Gerontologia. Pg. 354-360.

Junior, R. S. B.; \& Golias, A. R. C. (2021). Fraturas provocadas por acidentes de motocicleta. Maringá, PR. Revista Uningá, v. 58 , eUJ3756.

Machado, A. M.; Braga, A. L. F.; Garcia, M. L. B.; Martins, L. C. (2012). Avaliação da qualidade de vida em idosos pós-fratura da extremidade. Arquivos Brasileiros de Ciências da Saúde, v.37, n. 2, p. 70-75, Maio/Ago. 
Research, Society and Development, v. 11, n. 3, e4511325518, 2022

(CC BY 4.0) | ISSN 2525-3409 | DOI: http://dx.doi.org/10.33448/rsd-v11i3.25518

Maia, B. C.; Viana, P. S.; Arantes, P. M. M.; Alencar, M. A. (2011). Consequências das Quedas em Idosos Vivendo na Comunidade. Rev. Bras. Geriatr. Gerontol. Rio de janeiro - RJ.14(2):381-393.

Moraes, F. B.; Silva, L. L.; Ferreira, F. V.; Ferro, A. M.; Rocha, V. L.; Teixeira, K. S. (2009). Avaliação epidemiológica e radiológica das fraturas diafisárias do fêmur: estudo de 200 casos. Rev. bras. ortop. 44 (3) Jun.

Muniz, C. F.; Arnaut, A. C.; Yoshida, M.; Trelha, C. S. (2007). Caracterização dos idosos com fratura de fêmur proximal Atendidos em hospital escola público. Revista Espaço para a Saúde, Londrina, v.8, n.2, p.33-38.

Oliveira, M. C.; Rabelo, W. M.; Silva, M. C. P.; Faro, S. M. L.; Weekes, K. W.; Costa, G. L. C.; Vallinoto, I. M. V. C. (2020). Fraturas de fêmur em pacientes idosos e os serviços de saúde pública na região Amazônica. Research, Society and Development, [S. 1.], v. 9, n. 9, p. e701997725.

Peden, M. (2005). Global collaboration on road traffic injury prevention. Int. J. Inj. Contr. Saf. Promot. 12:85-91.

Pires, R. E. S.; Rei, F. B.; Simões, C. E.; Santos, L. E. N.; Rodrigues, V. B.; Andrade, M. A. P.; Neto, P. J. P. (2010). Fratura diafisária do fêmur: reprodutibilidade das classificações AO-ASIF e Winquist. Acta Ortop Bras. 18(4):197-9.

Rios, P. A. A.; Mota, E. L. A.; Ferreira, L. N.; Cardoso, J. P.; Santos, G. J.; Rodrigues, T. B. (2019). Acidentes de trânsito com condutores de veículos: incidência e diferenciais entre motociclistas e motoristas em estudo de base populacional. Rev. bras. epidemiol. 22.

Rocha, R. O.; Alkmim, L. R. F.; Siqueira, A. R.; Xavier, M. D.; Oliveira, S. P.; Bauman, C. D. (2020). Perfil epidemiológico das diferentes fraturas de fêmur de pacientes internados em um hospital do norte de Minas Gerais. Revista Eletrônica Acervo Saúde. Montes Claros MG. Vol.12(12)|e5753| Página 1 de 9.

Santos Júnior, J. E.; \& Silva, R. B. B. (2021). Fraturas do fêmur em idosos do Nordeste do Brasil: dados epidemiológicos e gastos para o SUS. Research, Society and Development, [S. 1.], v. 10, n. 14, p. e180101421984.

Santos, M. S.; Ferreira, C. F.; Ferreira, F. V.; Dall'agno, M. L.; Wender, M. C. O. (2021). Fatores associados a fraturas de fêmur em uma coorte de mulheres idosas. Research, Society and Development, [S. 1.], v. 10, n. 10, p. e145101018439.

Soares, D. S.; Mello, L. M.; Silva, A. S.; Martinez, E. Z.; Nunes, A. A. (2014). Fraturas de fêmur em idosos no Brasil: análise espaço-temporal de 2008 a 2012. Cad. Saúde Pública, Rio de Janeiro, 30(12):2669-2678.

Soares, D. S.; Mello, L. M.; Silva, A.; Nunes, A. A. (2015). Análise dos fatores associados a quedas com fratura de fêmur em idosos: um estudo caso-controle. Rev. Bras. Geriatr. Gerontol. Rio de Janeiro, 2015; 18(2):239-248. 